\title{
EXTENSIONS OF FUNCTIONS AND SPACES
}

\author{
BY \\ GIOVANNI VIGLINO
}

\begin{abstract}
We investigate, for a given map $\varphi$ from a topological space $X$ to a topological space $Y$ (denoted by $[X, \varphi, Y]$ ), those triples $[E, \Phi, Y]$ where $E$ is an extension of $X$ and $\Phi$ extends $\varphi$ to $E$. A maximal such extension, similar to the Katětov extension of a topological space, is examined.
\end{abstract}

Introduction. An extension of a topological space $X$ is a pair $(E, \psi)$ where $E$ is a topological space, $\psi$ is a homeomorphism of $X$ into $E$ and $\psi(X)$ is dense in $E$. Let $(E, \psi)$ be an extension of $X$ and let $\varphi$ denote a (continuous) map from $X$ into a space $Y$. A map $\Phi: E \rightarrow Y$ is, by definition, an extension of $\varphi$ if $\varphi(x)=\Phi \psi(x)$ for every $x \in X$. We shall investigate for given $\varphi: X \rightarrow Y$ (henceforth denoted by $[X, \varphi, Y])$ simultaneous extensions of both $X$ and $\varphi$, that is triples $[(E, \psi), \Phi, Y]$ where $(E, \psi)$ extends $X$ and $\Phi$ extends $\varphi$. Such a triple $[(E, \psi), \Phi, Y]$ will be called an extension of $[X, \varphi, Y]$. The study of extensions of a space $X$ is the study of extension of $[X, P,\{p\}]$ where $\{p\}$ is the one point space and $P$ is the map from $X$ to $\{p\}$. Since, for arbitrary $[X, \varphi, Y]$ we can at best extend $\varphi$ to $\overline{\varphi(X)}$, we shall assume throughout this discussion that $\varphi(X)$ is dense in $Y$. We further make the assumption that all spaces are Hausdorff.

\section{Extensions.}

Definition 1.1. Two extensions $[(E, \psi), \Phi, Y],\left[\left(E^{\prime}, \psi^{\prime}\right), \Phi^{\prime}, Y\right]$ of $[X, \varphi, Y]$ are equivalent $(=)$ if there exists a homeomorphism $\theta: E \rightarrow E^{\prime}$ such that

(i) $\Phi^{\prime} \theta=\Phi$ (on $E$ ) and

(ii) $\theta \psi=\psi^{\prime}$ (on $X$ ).

Let $X$ be a space and $x \in X$. $\mathcal{N}(x)$ will denote the open neighborhood system of $x$. We use a well-established technique (cf. [2], [4]) of constructing extensions of topological spaces to select a representative from each equivalence class of extensions of $[X, \varphi, Y]$. Let $[(E, \psi), \Phi, Y]$ extend $[X, \varphi, Y]$. Topologize $E^{\prime}=X$ $\cup\left\{\psi^{-1}(\mathcal{N}(e)) \mid e \in E \backslash \psi(X)\right\}$ so that the bijection $x \rightarrow \psi(x)$ for $x \in X$, and $\psi^{-1}(\mathcal{N}(e)) \rightarrow e$ is a homeomorphism. We denote the extension $\left(E^{\prime}, i\right)$ of $X$, where $i$ is the inclusion map on $X$, simply by $E^{\prime}$. Extend $\varphi$ to a map $\Phi^{\prime}$ on $E^{\prime}$ by defining $\Phi^{\prime}\left[\psi^{-1}(\mathcal{N}(e))\right]=\Phi(e)$. Then, $\left[E^{\prime}, \Phi^{\prime}, Y\right]=[(E, \psi), \Phi, Y]$. We shall therefore assume, unless otherwise stated, that an extension of $[X, \varphi, Y]$ is a triple $[E, \Phi, Y]$ where $E$ contains $X, E \backslash X$ consists of open filters on $X$, and $\xi \in E \backslash X$ implies

Presented to the Society, April 29, 1971; received by the editors May 4, 1971 and, in revised form, May 1, 1972.

AMS (MOS) subject classifications (1970). Primary 54C20, 54B99, 54Cl0.

Key words and phrases. Extension, maximal extension, Katětov extension, absolutely closed, perfect map, semiregular. 
that $\xi$ equals the trace of the open neighborhoods of $\xi$ on $X$.

In the case that $Y$ is regular, we can characterize all extensions of $[X, \varphi, Y]$ in the following way: Let $\mathcal{} M=\left\{\varphi^{-1} \mathcal{N}(y) \mid y \in Y\right\}$. Let $E=X \cup\left\{\xi_{\alpha}\right\}_{\alpha \in A}$ where $\xi_{\alpha}$ is a free open filter in $X$ containing an element of $\mathcal{M}$ for each $\alpha \in A$, and given distinct elements, $\alpha$ and $\alpha^{\prime}$, of $A$ then there exist $O_{\alpha} \in \xi_{\alpha}$ and $O_{\alpha}^{\prime} \in \xi_{\alpha^{\prime}}$ with $O_{\alpha} \cap O_{\alpha}^{\prime}=\varnothing$. Extend $\varphi$ to a function $\Phi$ on $E$ by defining $\Phi\left(\xi_{\alpha}\right)=y$ where $\varphi^{-1} \mathcal{N}(y) \subset \xi_{\alpha}$, for $\alpha \in A$. There are two "natural topologies" one can impose on $E$ (cf. [2]) for which [ $E, \Phi, Y]$ becomes an extension of $[X, \varphi, Y]$. These are the strict and the simple extension topologies, where the strict topology, $\tau_{0}$, is generated by the sets $O \cup\left\{\xi_{\alpha} \mid O \in \xi_{\alpha}\right\}$ for $O$ open in $X$, and the simple topology, $\tau_{1}$, is generated by the open sets in $X$ union the sets $\left\{\xi_{\alpha}\right\} \cup O$ where $O \in \xi_{\alpha}$, for $\alpha \in A$. Hence, for any topology $\tau$ on $E$ with $\tau_{0} \leq \tau \leq \tau_{1}$ we have that $[E, \Phi, Y]$ extends $[X, \varphi, Y]$. One can easily show that these extensions represent up to equivalence all possible extensions of $[X, \varphi, Y]$.

Definition 1.2. A space $(X, \tau)$ is semiregular if $\left\{\overline{O^{\circ}} \mid O \in \tau\right\}$ is a base for $\tau$.

Theorem 1.1. Let $[X, \varphi, Y]$ be given. Then there exists an extension $[E, \Phi, Y]$ with $\Phi(E)=Y$. In the case that $Y$ is semiregular, the extension may be chosen so that $E \backslash X$ is homeomorphic to $Y \backslash \varphi(X)$.

Proof. In the general case, let $E=X \cup\left\{\varphi^{-1} \mathcal{N}(y) \mid y \in Y \backslash \varphi(X)\right\}$ with simple topology and define $\Phi\left(\varphi^{-1} \mathcal{N}(y)\right)=y$. In the case that $Y$ is semiregular, choose the same set $E$ and function $\Phi$ used in the general case and give $E$ the strict topology. Clearly $\Phi$ is surjective and a bijection from $E \backslash X$ to $Y \backslash \varphi(X)$. $\Phi$ is continuous: Let $W$ be an open neighborhood of $y_{0} \in Y \backslash \varphi(X)$ and $V \subset W$ a neighborhood of $y_{0}$ with $V=\overline{V^{o}}$. Let $O=\varphi^{-1}(V) \cup\left\{\xi \in E \backslash X \mid \varphi^{-1}(V) \in \xi\right\}$. Clearly $y_{0} \in \Phi(O)$. Suppose $\xi \in O$ with $\xi=\varphi^{-1} \mathcal{N}(y), y \notin W$. Since $V=\overline{V^{o}}$ we have that $\overline{V^{o}} \cap U \neq \varnothing$ for each $U \in \mathcal{N}(y)$. It then follows, since $\varphi(X)$ is dense in $Y$, that $\varphi^{-1}(V) \notin \varphi^{-1} \mathcal{N}(y)$, contradicting the fact that $\xi \in O$. Hence, $\Phi(O) \subset W$ and $\Phi$ is continuous. Let $\Phi_{R}$ denote the restriction of the map $\Phi$ to $E \backslash X$. We complete the proof of the theorem by showing that $\Phi_{R}^{-1}$ is continuous. Let $\varphi^{-1} \mathcal{N}\left(y_{0}\right)$ with basic open neighborhood $U=\left\{\xi \in E \backslash X \mid \varphi^{-1}(V) \in \xi\right\}$ in $E \backslash X$ be given, for $V \in \mathcal{N}\left(y_{0}\right)$. Since $V \in \mathcal{N}(y)$ for each $y \in V \cap$ $[Y \backslash \varphi(X)]$ we have $\Phi_{R}^{-1}(V \cap[Y \backslash \varphi(X)]) \subset U$.

Definition 1.3. (a) A space $X$ is absolutely closed if there exists no proper extension of $X$.

(b) Let $[X, \varphi, Y]$ be given. $X$ is $\varphi$-absolutely closed if there exists no proper extension of $[X, \varphi, Y]$.

(c) Let $[X, \varphi, Y]$ be given. An open filter, $\xi$, in $X$ is $\varphi$-convergent if the filter $\varphi(\xi)$ converges in $Y$.

An immediate consequence of Theorem 1.1 is that a proper extension exists for a triple $[X, \varphi, Y]$ if $\varphi$ is not onto. In general, we cannot expect the existence of a proper extension for a triple $[X, \varphi, Y]$; certainly no such extension will exist in the case that $X$ is absolutely closed. 
Theorem 1.2. Let $[X, \varphi, Y]$ be given. $X$ is $\varphi$-absolutely closed if and only if every $\varphi$-convergent filter in $X$ has nonempty adherent set.

Proof. Let $\xi$ be a $\varphi$-convergent filter with empty adherent set $\varphi$-converging to $y$. Let $E=X \cup\{\xi\}$ with $X$ retaining its topology and with a neighborhood system of the point $\xi$ consisting of all sets of the form $\{\xi\} \cup O$ where $O \in \xi$. Extending $\varphi$ to $\Phi$ on $E$ by defining $\Phi(\xi)=y$ we have that $[E, \Phi, Y]$ extends $[X, \varphi, Y]$. Hence $X$ is not $\varphi$-absolutely closed.

Conversely, suppose $[E, \Phi, Y]$ is a proper extension. Let $\xi \in E \backslash X$. Since $E$ is Hausdorff, $\xi$ must be an open filter with no adherent point. Since $\Phi$ is continuous, $\xi$ must be $\varphi$-convergent.

The following example shows that $X$ may be $\varphi$-absolutely closed without being absolutely closed for given $[X, \varphi, Y]$. In the case that $Y$ is compact however, the properties absolutely closed and $\varphi$-absolutely closed are equivalent, as is shown in Corollary 1.1 below.

Example 1.1. Let $Z$ denote the open interval $(0,1)$ with the usual topology. Let $Y$ denote the same interval with topology generated by the usual open subsets and the rationals. Let $i$ denote the identity map from $Y$ to $Z$. Clearly $Y$ is not absolutely closed. It is however $i$-absolutely closed since any open filter in $Y$ containing $i^{-1} \mathcal{N}(r)$ must have $\{r\}$ as adherent set, for any $r \in(0,1)$.

Corollary 1.1. Let $[X, \varphi, Y]$ be given with $Y$ compact. Then $X$ is $\varphi$-absolutely closed if and only if $X$ is absolutely closed.

Proof. We need only prove the necessary part of the theorem. To do this we use the fact that a space $X$ is absolutely closed if (and only if) every maximal open filter in $X$ has nonempty adherent set [1]. Let $\xi$ denote a maximal open filter in $X$. Suppose $\xi$ is not $\varphi$-convergent. Then for each $y \in Y$ there exists an open neighborhood $O_{y}$ with $\varphi^{-1}\left(O_{y}\right) \notin \xi$. Let $Y=\cup_{i=1}^{n} O_{y_{i}}$. Since $\xi$ is maximal and since $\left\{\varphi^{-1}\left(O_{y_{i}}\right)\right\}_{i=1}^{n}$ covers $X$, some $\varphi^{-1}\left(O_{y_{i}}\right) \in \xi, 1 \leq i \leq n$, a contradiction. Therefore $\xi$ is $\varphi$-convergent and, by Theorem 1.2, $\xi$ has nonempty adherent set.

Clearly if $\varphi: X \rightarrow Y$ is a homeomorphism then $X$ is $\varphi$-absolutely closed. The converse, as shown by Example 1.1, does not in general hold. However, the converse is valid in the case that $X$ is semiregular and $\varphi$ is injective.

Corollary 1.2. Let $X$ be semiregular and $\varphi: X \rightarrow Y$ be injective. Then $X$ is $\varphi$ absolutely closed if and only if $\varphi$ is a homeomorphism.

Proof. We need only prove the necessary part of the theorem.

Suppose $\varphi$ is not a homeomorphism. Then there exists a $y \in Y$ such that $\varphi^{-1} \mathcal{N}(y)$ is not a neighborhood base for any $x \in X$. If $\varphi$ is not onto then $X$ is not $\varphi$-absolutely closed by Theorem 1.1. If $\varphi$ is onto then let $x$ be such that $\varphi(x)=y$. Choose a neighborhood $O=\bar{O}^{o}$ of $x$ which contains no element of $\varphi^{-1} \mathcal{N}(y)$. Then, $\varphi^{-1} \mathcal{N}(y) \cup \bar{O}^{c}$ is a $\varphi$-convergent filter with empty adherent set so that $X$ is not $\varphi$-absolutely closed. 
Definition 1.4. A map $\varphi: X \rightarrow Y$ is perfect if it is a continuous closed surjection with $\varphi^{-1}(y)$ compact for each $y \in Y$.

Theorem 1.3. Let $[X, \varphi, Y]$ be given with $X$ regular. Then, $\varphi$ is a perfect map if and only if $X$ is $\varphi$-absolutely closed.

Proof. It is well known that if $\varphi$ is perfect then every $\varphi$-convergent filter has nonempty adherent set, cf. [3, p. 254]. We show, in the case that $X$ is regular, that the converse is also valid. Let $X$ be $\varphi$-absolutely closed. By Theorem $1.1, \varphi$ is surjective. Suppose there exists a $y \in Y$ such that the fibre $\varphi^{-1}(y)$ is not compact. Then there exists, by regularity, an open cover $\mathcal{O}=\left\{O_{\alpha}\right\}_{\alpha \in A}$ of $\varphi^{-1}(y)$ such that the closure of any finite union of elements of $\mathcal{O}$ fails to contain $\varphi^{-1}(y)$. We may assume $\mathcal{O}$ to be closed under finite union. Then, $\varphi^{-1} \mathcal{N}(y) \cup\left\{\bar{O}_{\alpha}^{c}\right\}_{\alpha \in A}$ is a free filter, since any adherent point of a filter containing $\varphi^{-1} \mathcal{N}(y)$ must be in $\varphi^{-1}(y)$. The filter is therefore free and $\varphi$-convergent contradicting the assumption that $X$ is $\varphi$-absolutely closed. Finally, suppose $\varphi$ were not closed. Choose a closed subset $C$ of $X$ and an element $y$ such that $y \in \overline{\varphi(C)} \backslash C$. Since $\varphi^{-1}(y)$ is compact and $X$ is regular, we may choose an open set $O$ with $\varphi^{-1}(y) \subset O$ and $C \cap \bar{O}=\varnothing \cdot \varphi^{-1} \mathcal{N}(y) \cup\left\{\bar{O}^{c}\right\}$ is a free $\varphi$-convergent filter, contradicting the assumption that $X$ is $\varphi$-absolutely closed.

In general, if $\varphi$ is perfect then no extension exists since no $\varphi$-convergent (or indeed $\varphi$-adherent) filter is free. In the event that $X$ is not regular however, $X$ may be $\varphi$-absolutely closed with $\varphi$ not closed (see Example 1.1). Also, if $X$ is not regular, $X$ may be $\varphi$-absolutely closed with $\varphi^{-1}(y)$ not compact for some $y \in Y$. To see this one need only consider the triple $[X, \varphi,\{y\}]$ where $X$ is absolutely closed but not compact (see Corollary 1.1).

Definition 1.5. (a) A subset $S$ of a space $X$ is $X$-absolutely closed if for any cover $\mathcal{O}$ of $S$ by sets open in $X$ there exists a finite number of elements in $\mathcal{O}$, say $O_{1}, \ldots, O_{n}$ with $S \subset \mathrm{Cl}_{X} \cup_{i=1}^{n} O_{i}$.

(b) A map $\varphi: X \rightarrow Y$ is $H$-perfect if it is a continuous surjection with $\varphi^{-1}(y)$ $X$-absolutely closed for each $y \in Y$.

One may easily show that $S \subset X$ is $X$-absolutely closed if and only if $S$ is closed in any extension of $X$.

Applying part of the prof of the previous theorem one obtains the following theorem.

Theorem 1.4. Let $[X, \varphi, Y]$ be given ( $X$ not necessarily regular). If $X$ is $\varphi$ absolutely closed then $\varphi$ is $H$-perfect.

The converse of the above theorem is false. To see this, let $\varphi: X \rightarrow Y$ be a continuous surjection with $\varphi^{-1}(y)$ compact for each $y \in Y$. Assume further that $X$ is regular and $\varphi$ is not closed. Clearly $\varphi$ is $H$-perfect. However, by Theorem $1.3, X$ is not $\varphi$-absolutely closed.

Question. Let $[X, \varphi, Y]$ be given. Let $\varphi$ be $H$-perfect and closed. Is $X \varphi$ absolutely closed? 
Theorem 1.5. Let $[X, \varphi, Y]$ and $[Y, \gamma, Z]$ be given. If $X$ is $(\gamma \varphi)$-absolutely closed then $X$ must be $\varphi$-absolutely closed and $Y \gamma$-absolutely closed.

Proof. Suppose $X$ is not $\varphi$-absolutely closed. Then there exists $y \in Y$ and a free filter $\xi$ on $X$ which contains $\varphi^{-1} \mathcal{N}(y)$. Since $\xi$ then contains $(\gamma \varphi)^{-1} \mathcal{N}(\gamma(y))$ we have that $X$ is not $(\gamma \varphi)$-absolutely closed.

Suppose $Y$ is not $\gamma$-absolutely closed. Then there exists $z \in Z$ and a free filter $\xi$ on $Y$ which contains $\gamma^{-1} \mathcal{N}(z)$. Since $\varphi^{-1}(\xi)$ is a free filter on $X$ containing $(\gamma \varphi)^{-1} \mathcal{N}(z)$ we have that $X$ is not $(\gamma \varphi)$-absolutely closed.

In the case that $X$ and $Y$ are regular the above theorem reduces to the wellknown result that if $\gamma \varphi$ is a perfect map then both $\varphi$ and $\gamma$ must be perfect maps. The following example shows that even if both $X$ is $\varphi$-absolutely closed and $Y$ is $\gamma$-absolutely closed then $X$ need not be $(\gamma \varphi)$-absolutely closed. In such an example, both $X$ and $Y$ could not be regular since a composite of perfect maps is perfect.

Example 1.2. Let $P$ denote the plane with topology generated by the standard topology of the plane and the set of rational points in the plane. Let $X$ be the subspace $\{(x, 0) \mid x \in(0,1)\} \cup\{(x, 1) \mid x \in(0,1), x$ is irrational $\}$. Let $[Y, i, Z]$ be as in Example 1.1. Let $\pi$ denote the projection map from $X$ onto $Y$. As noted in Example 1.1, $Y$ is $i$-absolutely closed. Since for any $y \in Y$, any open filter in $X$ containing $\pi^{-1} \mathcal{N}(y)$ must contain $(y, 0)$ in its adherent set we have that $X$ is $\pi$ absolutely closed. $X$ is not $(i \pi)$-absolutely closed, for adjoining to the filter

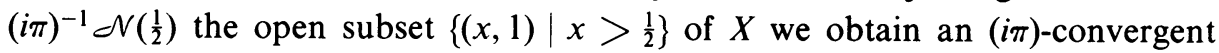
filter with no adherent point.

A consequence of the previous theorem is that for a given triple $[X, \varphi$, $\left.\prod_{\alpha \in A} Y_{\alpha}\right]$ if $X$ is $\left(\pi_{\alpha_{0}} \varphi\right)$-absolutely closed for some $\alpha_{0} \in A$, then $X$ is $\varphi$-absolutely closed. The following example shows that the converse does not hold.

Example 1.3. Let $Z$ denote the set of integers with discrete topology. Let $i$ denote the identity map on $Z \times Z$. By Corollary $1.2, Z \times Z$ is $i$-absolutely closed. $Z \times Z$ is not $\left(\pi_{1} i\right)$-absolutely closed. For let $\Im$ denote the set of finite complements of $\left\{\left(n_{0}, m\right) \mid m \in Z\right\}$. Then $\exists$ is a free open filter in $Z \times Z\left(\pi_{1} i\right)$-converging to $n_{0}$. Similarly $Z \times Z$ may be shown not to be $\left(\pi_{2} i\right)$-absolutely closed.

Theorem 1.6. Let $[X, \varphi, Y]$ be given and let $\left\{U_{\alpha}\right\}_{\alpha \in A}$ be an open cover for the space $Y$. Denote by $\varphi_{\alpha}$ the restriction of the map $\varphi$ to the subspace $\varphi^{-1}\left(U_{\alpha}\right)$ of $X$ onto the subspace $U_{\alpha}$ of $Y$. Then, $X$ is $\varphi$-absolutely closed if and only if $\varphi^{-1}\left(U_{\alpha}\right)$ is $\varphi_{\alpha}$ absolutely closed for each $\alpha \in A$.

Proof. Suppose $\varphi^{-1}\left(U_{\alpha}\right)$ is not $\varphi_{\alpha}$-absolutely closed. Let $\xi$ be a free open filter on $\varphi^{-1}\left(U_{\alpha}\right)$ which $\varphi_{\alpha}$-converges to $y$. Clearly $\xi_{0}=\xi \cup \varphi^{-1} \mathcal{N}(y) \varphi$-converges to $y$. If $x$ is adherent to $\xi_{0}$ in $X$ then $\varphi(x)=y$; therefore $x \in \varphi^{-1}\left(U_{\alpha}\right)$, and $x$ is adherent to $\xi$ in $\varphi^{-1}\left(U_{\alpha}\right)$. It follows that $\xi_{0}$ is free. Hence $X$ is not $\varphi$-absolutely closed.

Conversely, suppose $X$ is not $\varphi$-absolutely closed. Let $\xi$ be a free open filter in 
$X$ which $\varphi$-converges to $y \in U_{\alpha} . \xi \cap \varphi^{-1}\left(U_{\alpha}\right)$ is then a free open filter on $\varphi^{-1}\left(U_{\alpha}\right)$ which $\varphi_{\alpha}$-converges to $y$. Hence $\varphi^{-1}\left(U_{\alpha}\right)$ is not $\varphi_{\alpha}$-absolutely closed.

In the case that each $X_{\alpha}$ is regular, the following theorem is, by Theorem 1.3, the well-known fact that $\prod_{\alpha \in A} \varphi_{\alpha}$ is a perfect map if and only if each $\varphi_{\alpha}$ is a perfect map. In the case that each $Y_{\alpha}$ is a single point, the following theorem reduces to the well-known fact that a product space is absolutely closed if and only if each factor is absolutely closed [7].

Theorem 1.7. Let $\left\{\left[X_{\alpha}, \varphi_{\alpha}, Y_{\alpha}\right]\right\}_{\alpha \in A}$ be given. Then $\prod_{\alpha} X_{\alpha}$ is $\prod_{\alpha} \varphi_{\alpha}$-absolutely closed if and only if each $X_{\alpha}$ is $\varphi_{\alpha}$-absolutely closed.

Proof. Suppose each $X_{\alpha}$ is $\varphi_{\alpha}$-absolutely closed. Let $y=\left\{y_{\alpha}\right\}_{\alpha \in A} \in \prod_{\alpha} Y_{\alpha}$. Let $\xi$ be any open filter in $\prod_{\alpha} X_{\alpha}$ which is $\prod_{\alpha} \varphi_{\alpha}$-convergent to $y$. Then, for each $\alpha \in A, \pi_{\alpha}(\xi)$ is a $\varphi_{\alpha}$-convergent open filter in $X_{\alpha}$ and therefore contains an adherent point, say $x_{\alpha} .\left\{x_{\alpha}\right\}$ is then an adherent point of $\xi$.

Conversely, let $X_{\alpha_{0}}$ not be $\varphi_{\alpha_{0}}$-absolutely closed. Then there exists a free open filter, $\xi_{\alpha_{0}}$, with $\varphi_{\alpha_{0}}\left(\xi_{\alpha_{0}}\right)$ converging to $y_{\alpha_{0}} \in Y_{\alpha_{0}}$. Let $p=\left\{p_{\alpha}\right\}$ be any point in $\prod_{\alpha \neq \alpha_{0}} Y_{\alpha}$ and let $\xi$ denote the open filter in $\prod_{\alpha} X_{\alpha}$ generated by the sets

$$
\begin{aligned}
\left\{F_{\alpha_{0}} \times \prod_{\alpha \neq \alpha_{0}} Y_{\alpha} \mid F_{\alpha_{0}} \in \xi\right\} & \\
& \cup \underset{\alpha \neq \alpha_{0}}{\cup}\left\{\varphi_{\alpha}^{-1}(U) \times \prod_{\beta \in A ; \beta \neq \alpha} Y_{\beta} \mid U \text { is an open neighborhood of } p_{\alpha}\right\} .
\end{aligned}
$$

Then $\prod_{\alpha} \varphi_{\alpha}(\xi)$ converges to $y_{\alpha_{0}} \times p$ and is free.

2. Maximal extensions.

Definition 2.1. Let $[(E, \psi), \Phi, Y]$ and $\left[\left(E^{\prime}, \psi^{\prime}\right), \Phi^{\prime}, Y\right]$ be extensions of $[X, \varphi, Y]$. $[(E, \psi), \Phi, Y]$ is not less than $(\geq)\left[\left(E^{\prime}, \psi^{\prime}\right), \Phi^{\prime}, Y\right]$ if there exists a continuous surjection $\theta$ from a subspace $S$ of $E$ containing $\psi(X)$ to $E^{\prime}$ such that

(i) $\Phi^{\prime} \theta=\Phi($ on $S)$ and

(ii) $\theta \psi=\psi^{\prime}$ (on $X$ ).

Considering two equivalent extensions as being equal we have that the relation $\geq$ is a partial order on the set of extensions of $[X, \varphi, Y]$. That the collection is a set follows from our restriction of extensions of $X$ to Hausdorff extensions. That the relation is a preorder is immediate. To see that the relation is a partial order one need only apply the following lemma.

Lemma. Let $X$ be dense in the spaces $E$ and $E^{\prime}$. Let $S$ and $S^{\prime}$ be subspaces of $E$ and $E^{\prime}$ containing $X$. Let $\theta$ be a map from $S$ onto $E^{\prime}$ which leaves $X$ fixed and let $\theta^{\prime}$ be a map from $S^{\prime}$ onto $E$ which leaves $X$ fixed. Then $\theta$ is a homeomorphism between $E$ and $E^{\prime}$. 
Proof. We need only show $S=E$ and $S^{\prime}=E^{\prime}$. Let $x \in E$. Choose $y \in S^{\prime}$ such that $\theta^{\prime}(y)=x$. Choose $s \in S$ with $\theta(s)=y$. The hypotheses of the lemma imply $x=s$.

Question. Is the set of extensions of $[X, \varphi, Y]$ a lattice, and if so, a complete lattice?

Clearly for given $[X, \varphi, Y]$ there is, up to equivalence, a smallest extension, namely $[X, \varphi, Y]$. For a given space $X$, Katětov [5] has constructed a maximal absolutely closed extension $K[X]=X \cup \mathcal{N}$ where $\mathcal{N}$ denotes the set of maximal free open filters, and the topology on $K[X]$ is the simple topology. We now generalize this construction.

Let $[X, \varphi, Y]$ be given. Let $K(\varphi)=X \cup \propto M$ with simple topology, where $\mathcal{M}$ denotes the set of maximal $\varphi$-convergent open filters with empty adherent set. Extending $\varphi$ to a map $\varphi^{*}$ on $K(\varphi)$ by defining $\varphi^{*}(\xi)=y$ where $\varphi^{-1} \mathcal{N}(y) \subset \xi$ we have that $\left[K(\varphi), \varphi^{*}, Y\right]$ is an extension of $[X, \varphi, Y]$. The fact that $K(\varphi)$ is Hausdorff follows from the maximality of the elements of $\mathcal{M}$ and from the fact that elements of $\mathcal{M}$ have empty adherent set. We show in the following theorem that $\left[K(\varphi), \varphi^{*}, Y\right]$ is greater than or equal to any extension of $[X, \varphi, Y]$. Since the set of extensions is partially ordered we have that $\left[K(\varphi), \varphi^{*}, Y\right]$ is characterized, up to equivalence, as the greatest extension.

Theorem 2.1. Let $[E, \Phi, Y]$ be any extension of $[X, \varphi, Y]$. Then $\left[K(\varphi), \varphi^{*}, Y\right]$ $\geq[E, \Phi, Y]$.

Proof. Let $E=X \cup \Im$ where $\exists$ consists of $\varphi$-convergent free filters. For

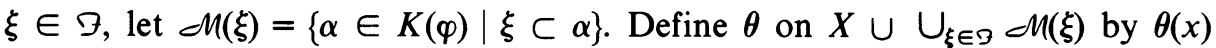
$=x$ for $x \in X$ and $\theta(\alpha)=\xi$ where $\xi \subset \alpha$ for $\alpha \in \cup_{\xi \in \mathcal{g}} \mathcal{M ( \xi ) . \theta}$ is well defined since, by the Hausdorff property of $E$, an element of $\bigcup_{\xi \in \mathcal{I}} \mathcal{} M(\xi)$ cannot contain two elements of $\Im$. The continuity of $\theta$ follows from the fact that $K(\varphi)$ is a simple extension. Clearly $\theta$ is a surjection and satisfies properties (i) and (ii) of Definition 2.1.

Let $\varphi$ be a map on a space $X$ and let $E$ be a subspace of $X$. The restriction of $\varphi$ to $E$ will be denoted by $\varphi_{E}$.

Theorem 2.2. Let $[X, \varphi, Y]$ be given and $X \subset E \subset K(\varphi)$. Then $\left[K\left(\varphi_{E}^{*}\right),\left(\varphi_{E}^{*}\right)^{*}, Y\right]$ $=\left[K(\varphi), \varphi^{*}, Y\right]$, where $\left[K(\varphi), \varphi^{*}, Y\right]$ is here considered as an extension of $\left[E, \varphi_{E}^{*}, Y\right]$. In particular, we have $\left[K(\varphi), \varphi^{*}, Y\right]=\left[K\left(\varphi^{*}\right), \varphi^{* *}, Y\right]$ so that $K(\varphi)$ is $\varphi^{*}$-absolutely closed.

Proof. Any extension $[Z, \gamma, Y]$ of $\left[E, \varphi_{E}^{*}, Y\right]$ is also an extension of $[X, \varphi, Y]$. For the map $\theta: S \rightarrow Z$ showing that $\left[K(\varphi), \varphi^{*}, Y\right] \geq[Z, \gamma, Y]$ in the proof of 2.1 one has $E \subset S$, and $\theta$ maps $E$ identically. Hence $\left[K(\varphi), \varphi^{*}, Y\right]$ is also the greatest extension of $\left[E, \varphi_{E}^{*}, Y\right]$.

Remark. Let $[X, \varphi, Y]$ and $[Y, \gamma, Z]$ be given. Since $\xi \in K(\varphi) \backslash X$ implies $\xi$ is a maximal free open filter on $X$ which contains $\varphi^{-1} \mathcal{N}(y)$ for some $y \in Y$, then $\xi$ contains $(\gamma \varphi)^{-1} \mathcal{N}(\gamma(y))$, so that $\xi \in K(\gamma \varphi)$. Hence, $K(\varphi) \subset K(\gamma \varphi)$. The surjec- 
tion $\varphi^{*}: K(\varphi) \rightarrow Y$ will be denoted by $\varphi_{\gamma}$ when we consider it as a map from $K(\varphi)$ into $K(\gamma)$. Though $K(\varphi)$ is $\varphi^{*}$-absolutely closed, it need not be $\varphi_{\gamma}$-absolutely closed; indeed, by Theorem 1.1, $K(\varphi)$ is $\varphi_{\gamma}$-absolutely closed if and only if $Y$ is $\gamma$-absolutely closed. By definition, $\left[K(\gamma \varphi),(\gamma \varphi)^{*}, Z\right]$ is the $K$-absolute closure of $[X, \gamma \varphi, Z]$. By the previous theorem it is also the $K$-absolute closure of $\left[K(\varphi), \gamma \varphi^{*}, Z\right]$ and of $\left[K(\varphi), \gamma^{*} \varphi_{\gamma}, Z\right]$. We show it also to be the $K$-absolute closure of $\left[K\left(\varphi_{\gamma}\right), \gamma^{*} \varphi_{\gamma}^{*}, Z\right]$ by showing this last triple to be equivalent to an extension $\left[E,(\gamma \varphi)_{E}^{*}, Z\right]$ of $\left[K(\varphi), \gamma \varphi^{*}, Z\right]$ for $K(\varphi) \subset E \subset K(\gamma \varphi)$ and then applying the previous theorem. Let $E=K(\varphi) \cup$ trace on $X$ of $\xi$ for $\xi \in K\left(\varphi_{1}\right) \backslash K(\varphi)$. Clearly $E \subset K(\gamma \varphi)$ and one can show that the map $\theta$ which leaves the points of $K(\varphi)$ fixed and takes $\xi \in K\left(\varphi_{1}\right) \backslash K(\varphi)$ into the trace on $X$ of $\xi$ satisfies the conditions of Definition 1.1.

We have established the following commutative diagram, where $i$ denotes the inclusion map. It is complete in the sense that the $K$-absolute closure of any triple in the diagram is represented in the diagram.

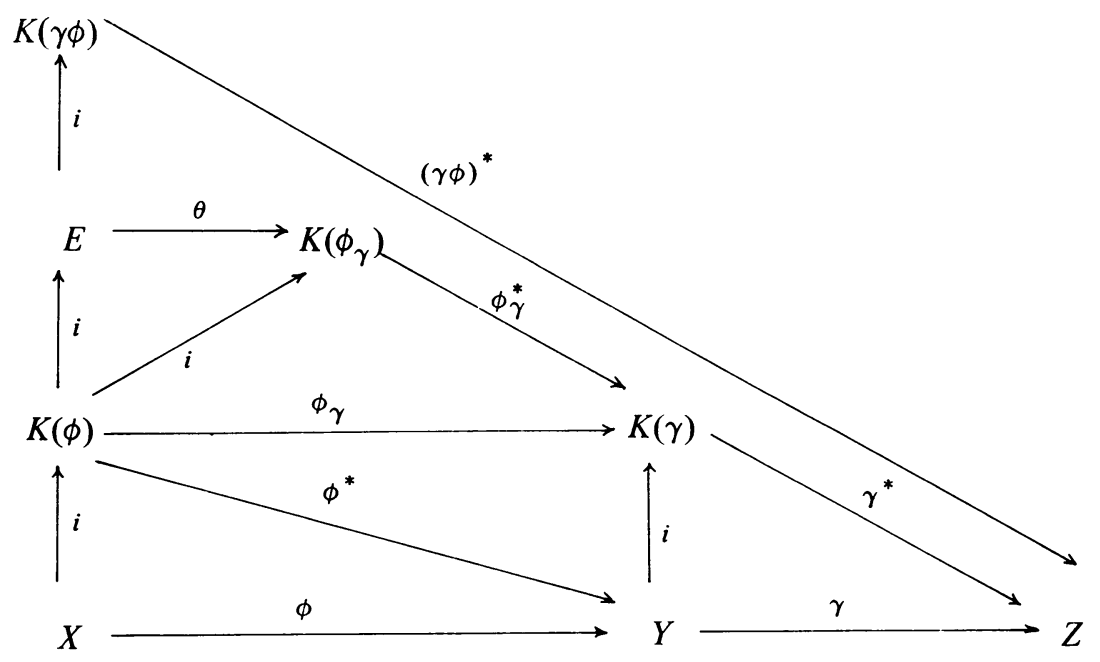

Let $\left\{\left[X_{\alpha}, \varphi_{\alpha}, Y_{\alpha}\right]\right\}_{\alpha \in A}$ be given and consider $\left[\prod_{\alpha} X_{\alpha}, \prod_{\alpha} \varphi_{\alpha}, \prod_{\alpha} Y_{\alpha}\right]$. If each $X_{\alpha}$ is $\varphi_{\alpha}$-absolutely closed then, by Theorem 1.7 , we have $K\left(\prod_{\alpha} \varphi_{\alpha}\right)=\prod_{\alpha} K\left(\varphi_{\alpha}\right)$ $=\prod_{\alpha} X_{\alpha}$. In general, equality does not hold. For example, in the case that each $Y_{\alpha}$ is a single point then $K\left(\varphi_{\alpha}\right)=K\left(X_{\alpha}\right)=$ the Katětov absolute closure of $X_{\alpha}$ and $K\left(\prod_{\alpha} \varphi_{\alpha}\right)=K\left(\prod_{\alpha} X_{\alpha}\right)=$ the Katětov absolute closure of $\prod_{\alpha \in A} X_{\alpha}$. The following theorem gives necessary and sufficient conditions for $\prod_{\alpha} K\left(\varphi_{\alpha}\right)=K\left(\prod_{\alpha} \varphi_{\alpha}\right)$ in this special case.

Theorem 2.3 (Liu [6]). Let $X_{\alpha}$ be nonempty spaces for $\alpha \in A$. Then $K\left(\prod_{\alpha} X_{\alpha}\right)$ $=\prod_{\alpha} K\left(X_{\alpha}\right)$ iff at least one of the following two conditions is satisfied.

(a) $X_{\alpha}$ is absolutely closed for each $\alpha \in A$. 
(b) There exists $X_{\alpha_{0}}$ which is not absolutely closed. $X_{\alpha}$ is finite for all $\alpha \neq \alpha_{0}$. Moreover, all but finitely many $X_{\alpha}$ 's have only one point.

Using arguments similar to those appearing in [6] one may generalize the above theorem to the following result .

Theorem 2.4. Let $X_{\alpha}$ be nonempty spaces for $\alpha \in A$. Then $K\left(\prod_{\alpha} \varphi_{\alpha}\right)$ $=\prod_{\alpha} K\left(\varphi_{\alpha}\right)$ iff at least one of the following two conditions are satisfied.

(a) $X_{\alpha}$ is $\varphi_{\alpha}$-absolutely closed for each $\alpha \in A$.

(b) There exists $X_{\alpha_{0}}$ which is not $\varphi_{\alpha_{0}}$-absolutely closed. $X_{\alpha}$ is finite for all $\alpha \neq \alpha_{0}$. Moreover, all but finitely many $X_{\alpha}$ 's have only one point.

The author wishes to express his gratitude to the referee for his valuable suggestions. In particular, the above theorem appears in answer to a question posed by the referee. Theorem 1.1 was first proved for the case that $Y$ is regular and then generalized to the case that $Y$ is semiregular as a result of a question posed by the referee. The question concerning the lattice structure of extensions is the referee's. Finally, the observation that $S \subset X$ is $X$-absolutely closed if and only if $S$ is closed in any extension of $X$ is due to the referee.

\section{REFERENCES}

1. P. S. Alexandroff and P. S. Urysohn, Mémoire sur les espaces topologiques compacts, Verh. Kon. Akad. Wetensch. Amsterdam. Afd. Natuurk. (1) 14 (1929), no. 1, 1-96.

2. B. Banaschewski, Extensions of topological spaces, Canad. Math. Bull. 7 (1964), 1-22. MR 28 \#4501.

3. J. Dugundji, Topology, Allyn and Bacon, Boston, Mass., 1966. MR 33 \# 1824.

4. S. Fomin, Extensions of topological spaces, Ann. of Math. (2) 44 (1943), 471-480. MR 5, 45.

5. M Katětov, Über H-abgeschlossene und bikompakte Räume, Casopis Pěst. Mat. Fys. 69 (1940), 36-49. MR 1, 317.

6. C.-T. Liu, Absolutely closed spaces, Trans. Amer. Math. Soc. 130 (1968), 86-104. MR 36 \# 2107.

7. F. Obreanu, Absolutely closed spaces, Acad. Rep. Pop. Române Bul. Sti. Ser. Mat. Fiz. Chim. 2 (1950), 21-25. MR 13, 483.

Department of Mathematics, Wesleyan University, Middletown, Connecticut 06457

Current address: Universidad de Oriente, Cumaná, Venezuela 